\title{
A channel Brownian pump powered by an unbiased external force
}

\author{
Bao-quan Ai1* and Liang-gang Liu $^{2}$ \\ 1 Institute for Condensed Matter Physics, \\ School of Physics and Telecommunication Engineering \\ and Laboratory of Photonic Information Technology, \\ South China Normal University, 510006 Guangzhou, China \\ 2 Faculty of Information Technology, \\ Macau University of Science and Technology, Macao
}

(Dated: October 31, 2018)

\begin{abstract}
A Brownian pump of particles in an asymmetric finite tube is investigated in the presence of an unbiased external force. The pumping system is bounded by two particle reservoirs. It is found that the particles can be pumped through the tube from a reservoir at low concentration to one at the same or higher concentration. There exists an optimized value of temperature (or the amplitude of the external force) at which the pumping capacity takes its maximum value. The pumping capacity decreases with increasing the radius at the bottleneck of the tube.

PACS numbers: 05. 06. Cd, 02. 50. Ey, 05. 40. Jc, 66. 10. Cb
\end{abstract}

Keywords: Channel Brownian pump, particle current, concentration ratio 


\section{INTRODUCTION}

Noisy transport far from equilibrium play a crucial role in many processes from physical and biological to social systems. Molecular motors are the paradigm of how to extract useful mechanical energy in the random environment of thermal fluctuations $\mathbf{1}^{\underline{\underline{3}}} \underline{\underline{3}}$. Inspired on Feynman's ratchet and pawl machine, the appearance of directed motion enhanced by thermal fluctuations has been named after the ratchet effect ${ }^{4}$.

The idea of applying the ratchet mechanism to model pumps has already appeared in the literature $\mathrm{e}^{-11}$. Prost and co-workers ${ }^{5}$ studied the transport of an asymmetric pump with a simple two-level model and quantified how vectorial symmetry plus dissipation creates a macroscopic motion, even in the absence of any externally applied gradient. Astumian and Derenyi ${ }^{6}$ investigated a chemically driven molecular electron pump in which charge can be pumped through a tiny gated portal from a reservoir at low electrochemical potential to on at the same or higher electrochemical potential by cyclically modulating the portal and gate energies. Kosztin and Schulten ${ }^{7}$ studied the fluctuation-driven molecular transport through an asymmetric potential pump and three transport mechanisms: driven by potential gradient, by an external periodic force and by nonequilibrium fluctuations. Nonadiabatic electron heat pump was investigated by Rey and coworkers ${ }^{8}$. They presented a mechanism for extracting heat metallic conductors based on the energy-selective transmission of electrons through a spatially asymmetric resonant structure subject to ac driving. Wambaugh and co-workers 9 studied the transport of fluxons in superconductors by alternating current rectification. They found that at nonzero temperatures, the asymmetric geometry of the ratchet sawteeth automatically converts applied ac inputs into a net de motion of fluxons. In their another work ${ }^{10}$, they studied how inertia and particle pair interactions can induce transverse rectification of particle flows driven through square arrays or chains of triangular defects perpendicularly to their symmetry axis. Recently, Sancho and Gomez-marin ${ }^{11}$ presented a model for a Brownian pump powered by a flashing ratchet mechanism. The pumping device was embedded in a finite region and bounded by particle reservoirs. Their emphasis is on finding what concentration gradient the pump can maintain.

The previous works on pump were on the consideration of the energy barriers. The present work is extend to the study of the molecular pump to the case of the entropic barriers. The entropic barriers may appear when coarsening the description of a complex system for 
simplifying its dynamics $12-14$. We emphasize on finding how the Brownian particles can be pumped through an asymmetric tube from a particle reservoir at low concentration to one at the same or higher concentration in the presence of an unbiased external force. Our other task is on investigation what factors can affect the pumping capacity.

\section{GENERAL ANALYSIS}

We consider overdamped Brownian particles moving in an asymmetric finite tube [Fig. 1] in the presence of an unbiased external force. The tube is embedded in a finite region and bounded by two particle reservoirs. Its overdamped dynamics is described by the following Langevin equations $\frac{12,15}{15}$

$$
\begin{gathered}
\gamma \frac{d x}{d t}=F(t)+\xi_{x}(t), \\
\gamma \frac{d y}{d t}=\xi_{y}(t), \\
\gamma \frac{d z}{d t}=\xi_{z}(t),
\end{gathered}
$$

where $x, y, z$ are the three-dimensional (3D) coordinates, $\gamma$ is the friction coefficient of the particle. $\xi_{x, y, z}(t)$ are the uncorrelated Gaussian white noises with zero mean and correlation function: $<\xi_{i}(t) \xi_{j}\left(t^{\prime}\right)>=2 \gamma k_{B} T \delta_{i, j} \delta\left(t-t^{\prime}\right)$ for $i, j=x, y, z$. $T$ is the absolute temperature. $k_{B}$ is the Boltzmann constant. $<\ldots>$ denotes an ensemble average over the distribution

of noise. Imposing reflecting boundary conditions in the transverse direction ensures the confinement of the dynamics within the tube. The shape of the tube is described by its radius,

$$
\omega(x)=a \sin \left(\frac{2 \pi x}{L}\right)+b, x_{0} \leq x \leq x_{0}+L,
$$

where $a$ is the parameter that controls the flat degree of the tube of the tube, $L$ is the length of the tube. The radius at the bottleneck is $r_{b}=b-a . x_{0}$ is the coordinate of the left end. $F(t)$ is an unbiased external force and satisfies

$$
F(t)=\left\{\begin{array}{l}
F_{0}, \quad n \tau \leq t<n \tau+\frac{1}{2} \tau \\
-F_{0}, \quad n \tau+\frac{1}{2} \tau<t \leq(n+1) \tau,
\end{array}\right.
$$

where $\tau$ is the period of the unbiased force and $F_{0}$ is its magnitude. 
When the particles move in a confined media, its movement equation can be described by the Fick-Jacobs equation $\underline{12} \underline{\underline{15}}$ which is derived from the 3D (or 2D) Smoluchowski equation after elimination of $y$ and $z$ coordinates by assuming equilibrium in the orthogonal directions. The complicated boundary conditions of the diffusion equation in irregular channels can be greatly simplified by introducing an entropic potential that accounts for the reduced space accessible for diffusion of the Brownian particle. Reduction of the coordinates may involve the appearance of the entropic barriers and an effective diffusion coefficient. When $\left|\omega^{\prime}(x)\right|<1$, the effective diffusion coefficient reads $\underline{12} \underline{\underline{15}}$

$$
D(x)=\frac{D_{0}}{\left[1+\omega^{\prime}(x)^{2}\right]^{\alpha}},
$$

where $D_{0}=k_{B} T / \gamma$ and $\alpha=1 / 2$ for three dimensions. The prime stands for the derivative with respect to the space variable $x$.

Consider the effective diffusion coefficient and the entropic barriers, the dynamics of a Brownian particle moving along the axis of the $3 \mathrm{D}$ tube can be described by the equation $\underline{12,15}$

$$
\frac{\partial \rho(x, t)}{\partial t}=\frac{\partial}{\partial x}\left[D(x) \frac{\partial \rho(x, t)}{\partial x}+\frac{D(x)}{k_{B} T} A^{\prime}(x, t) \rho(x, t)\right]=-\frac{\partial j(x, t)}{\partial x},
$$

where a free energy $A(x, t)=E-T S=-F(t) x-T k_{B} \ln h(x)$ is defined $\underline{12}-\underline{14}$ : here $E=$ $-F(t) x$ is the energy, $S=k_{B} \ln h(x)$ is the entropy, $h(x)$ is the dimensionless transverse cross section $\pi[\omega(x) / L]^{2}$ of the tube in three dimensions. $j(x, t)$ is the probability current and $\rho(x, t)$ is the particle concentration.

If $F(t)$ changes very slowly with respect to $t$, namely, its period is longer than any other time scale of the system, there exists a quasistatic state. In the steady state, the concentration is just a function of space thus the flux becomes a constant $j$. The concentration $\rho(x)$ follows a first order non homogeneous linear differential equation, whose formal solution is

$$
\rho(x)=\exp \left[-\int_{x_{0}}^{x} \frac{A^{\prime}(z)}{k_{B} T} d z\right]\left\{c_{0}-j \int_{x_{0}}^{x} \frac{d z}{D(z)} \exp \left[\int_{x_{0}}^{z} \frac{A^{\prime}(y)}{k_{B} T} d y\right]\right\} .
$$

Though, unlike typical Brownian motors, the boundary conditions are not periodic nor the normalized condition is imposed, the unknown constant $c_{0}$ can be found by imposing the left reservoir concentration $\rho_{0} \equiv \rho\left(x_{0}\right)$ and the right concentration $\rho_{1} \equiv \rho\left(x_{0}+L\right)$ as fixed boundary conditions ${ }^{11}$. Then $c_{0}=\rho\left(x_{0}\right)$ and

$$
j\left(F_{0}\right)=\frac{k_{B} T\left[\rho_{0}-\rho_{1} e^{-\frac{F_{0} L}{k_{B} T}}\right]}{\int_{x_{0}}^{x_{0}+L}\left[1+\omega^{\prime}(x)^{2}\right]^{\alpha} e^{-\frac{F_{0}\left(x-x_{0}\right)}{k_{B} T}}\left[\frac{\omega\left(x_{0}\right)}{\omega(x)}\right]^{2} d x} .
$$


The average current is

$$
J=\frac{1}{\tau} \int_{0}^{\tau} j(F(t)) d t=\frac{1}{2}\left[j\left(F_{0}\right)+j\left(-F_{0}\right)\right] .
$$

For studying the pumping capacity, we consider the situation in which $J$ tends to zero which corresponds the case in which the pump in maintaining the maximum concentration difference between the two reservoirs across the membrane with no net leaking of particle. The method is the same that in Ref. 11. This situation is analogous the stalling force in Brownian motors.

From Eqs. (8-10), we can obtain

$$
\frac{\rho_{1}}{\rho_{0}}=\frac{\int_{x_{0}}^{x_{0}+L}\left[1+\omega^{\prime}(x)^{2}\right]^{\alpha}\left[e^{\frac{F_{0}\left(x-x_{0}\right)}{k_{B} T}}+e^{-\frac{F_{0}\left(x-x_{0}\right)}{k_{B} T}}\right]\left[\frac{\omega\left(x_{0}\right)}{\omega(x)}\right]^{2} d x}{e^{-\frac{F_{0} L}{k_{B} T}} \int_{x_{0}}^{x_{0}+L}\left[1+\omega^{\prime}(x)^{2}\right]^{\alpha} e^{\frac{F_{0}\left(x-x_{0}\right)}{k_{B} T}}\left[\frac{\omega\left(x_{0}\right)}{\omega(x)}\right]^{2} d x+e^{\frac{F_{0} L}{k_{B} T}} \int_{x_{0}}^{x_{0}+L}\left[1+\omega^{\prime}(x)^{2}\right]^{\alpha} e^{-\frac{F_{0}\left(x-x_{0}\right)}{k_{B} T}}\left[\frac{\omega\left(x_{0}\right)}{\omega(x)}\right]^{2} d x} .
$$

\section{RESULTS AND DISCUSSIONS}

For simplicity, we take $k_{B}=1, \gamma=1$ and $L=2 \pi$ throughout the study. Our method is valid only when $\left|\omega^{\prime}(x)\right|<1$ and its validity is testified by using numerical simulations ${ }^{16}$. Thus, we take $a=\frac{1}{2 \pi}$ and $\left|\omega^{\prime}(x)\right|<<1$ which ensure the validity of our method.

\section{A. Current $J$ at $\rho_{1}=\rho_{0}$}

In order to check the effect of the magnitude for the tube, we plot the current $J$ as a function of period numbers $N$ ( we have $N$ cells in the channel) in Fig. 2. It is found that the current $J$ is independent of the period numbers $N$. Each cell has the same structure and pumping capacity. Thus, our results are not finite size effects.

Figure 3(a) shows the current as a function of the initial coordinate $x_{0}$ for different values of $F_{0}$ at $\rho_{0}=\rho_{1}$. In this case, the transport behavior is similar to that in a periodic tube. The current is positive for $0 \leq x_{0}<\frac{\pi}{2}$ and $\frac{3}{2} \pi<x_{0} \leq 2 \pi$, zero at $x_{0}=\frac{\pi}{2}$ and $\frac{3}{2} \pi$, and negative for $\frac{\pi}{2}<x_{0}<\frac{3}{2} \pi$. The positions of the negative peak and positive peak are determined by the parameters of the system. In order to illustrate the transport behavior, currents $j\left(F_{0}\right),-j\left(F_{0}\right)$ and $J$ versus $x_{0}$ for $F_{0}=0.5$ are shown in Fig. $3(\mathrm{~b})$. When $0 \leq x_{0}<\frac{\pi}{2}$ and 
$\frac{3}{2} \pi<x_{0} \leq 2 \pi, j\left(F_{0}\right)$ is more than $-j\left(-F_{0}\right)$, so the current $J$ is positive. When $\frac{\pi}{2}<x_{0}<\frac{3}{2} \pi$, $-j\left(-F_{0}\right)$ is more than $j\left(F_{0}\right)$ and $J$ is negative. $j\left(F_{0}\right)$ is equal to $-j\left(-F_{0}\right)$ at $x_{0}=\frac{\pi}{2}$ and $\frac{3}{2} \pi$.

In fact, the sign of the current is determined by the asymmetry of the tube. The corresponding shape of the tube is given by Fig. 4 . It is obvious that the initial coordinate $x_{0}$ determines the asymmetry of the tube. When the left space of the tube is bigger than the right $\left(x_{0}=0\right)$, the particles run to the right in the presence of the unbiased external force. The particles move to the left when the right space is bigger $\left(x_{0}=\pi\right)$. There is no current for the symmetric tube $\left(x_{0}=\frac{\pi}{2}, \frac{3}{2} \pi\right)$. Hence, the system can pump the particles from the left to the right for $0 \leq x_{0}<\frac{\pi}{2}$ and $\frac{3}{2} \pi<x_{0} \leq 2 \pi$. We take $x_{0}=0$ in the rest study.

\section{B. Ratio $\rho_{1} / \rho_{0}$ at $J=0$}

We now focus on the maximum concentration ratio at both end of the tube. The analytical results are given by Eq. (11). In Figs. 5-7, we investigate the pumping capacity and see how the concentration ratio depends on temperature $T$, the radius $r_{b}$ at the bottleneck and the amplitude $F_{0}$ of the external force.

Figure 5 presents the concentration ratio $\rho_{1} / \rho_{0}$ as a function of temperature $T$ at $x_{0}=0$. The curve is observed to be bell shaped. When $T \rightarrow 0$, the particles cannot reach all space of the tube and the effect of the asymmetric entropic barrier disappears and there is no pumping capacity $\left(\rho_{1} / \rho_{0}=1\right)$. When $T \rightarrow \infty$, the effect of the unbiased external force disappears and the pumping capacity goes to zero, also. Therefore, there is an optimized value of $T$ at which the ratio takes its maximum value, which indicates that the thermal noise can facilitate the particles pumping.

The ratio $\rho_{1} / \rho_{0}$ as a function of the radius $r_{b}$ at the bottleneck is plotted in Fig. 6 . When $r_{b} \rightarrow 0$, no particle can pass through the tube, thus, $\rho_{1} / \rho_{0} \rightarrow \infty$. With increasing of the radius $r_{b}$, the effect of the asymmetric entripic barrier reduces and the pumping capacity decreases.

Figure 7 shows the ratio $\rho_{1} / \rho_{0}$ vs the amplitude $F_{0}$ of the unbiased external force. When $F_{0} \rightarrow 0$, only the effect of the asymmetric entropic barrier exists, so the system has no pumping capacity. The ratio $\rho_{1} / \rho_{0}$ saturates to 1 in the large amplitude $F_{0}$ limit. Therefore, the pumping capacity is maximum when $F_{0}$ takes an optimized value. 


\section{Current $J$ at $\rho_{1}>\rho_{0}$}

In Fig. 8, the current $J$ versus temperature $T$ is presented for different values of $\rho_{1}$ at $\rho_{0}=1$. For lower values of $\rho_{1}$ the current is larger. When $\rho_{1}>\rho_{0}$, the current is negative for both too low or too high temperature. When $T \rightarrow 0$, the effect of the asymmetric entropic barrier disappears, the concentration difference dominates the transport, thus, the current is negative. When $T \rightarrow \infty$, the effect of the unbiased external force disappears, the transport is dominated by the concentration difference and the particles move to the left. If the concentration difference exceed its maximum value, the current will be always negative.

Figure 9 shows the current $J$ as a function of the radius $r_{b}$ at $\rho_{0}=1, \rho_{1}=4$. The curve is observed to be bell shaped. When $r_{b} \rightarrow 0$, the tube is jammed and the particles cannot pass through the tube. When $r_{b} \rightarrow \infty$, the effect of the asymmetric entropic barrier disappears, and the current is dominated by the concentration difference, thus the current is negative. So there exists an optimized value of $r_{b}$ at which the current takes its maximum value.

\section{CONCLUDING REMARKS}

In this study, we study the transport of Brownian particles moving in an asymmetric finite tube in the presence of an unbiased external force. The pumping device is embedded in a finite region and bounded by two particle reservoirs. It is found that the particles can be pumped from low concentration reservoir to higher concentration reservoir. When $\rho_{1}=\rho_{0}$, the current is positive for $0<x_{0}<\pi / 2$ and $3 \pi / 2<x_{0}<2 \pi$, zero at $x_{0}=\pi / 2$ and $3 \pi / 2$, negative for $2 \pi<x_{0}<3 \pi / 2$. The sign of the current is determined by the asymmetry of the tube. The maximum ratio of the concentrations $(J=0)$ at both ends of the tube is also studied. We can find that there is an optimized value of temperature $T$ (or the amplitude $F_{0}$ of the external force) which gives the maximum ratio of $\rho_{1} / \rho_{0}$. The ratio $\rho_{1} / \rho_{0}$ decreases with increasing the radius $r_{b}$ at the bottleneck of the tube. We also study the current $J$ as a function of $J$ and $r_{b}$. We observe that the current can tend to its maximum value when $T$ or $r_{b}$ take the optimized value.

Though the model presented does not pretend to be a realistic model for a biological pumps, the results we have presented have a wide application in many systems, such as Na, K-ATPase pumps ${ }^{17}$, membrane proteins ${ }^{18}$, and electron pumps ${ }^{19}$. 


\section{ACKNOWLEDGMENTS}

The work was supported by the National Natural Science Foundation of China under Grant No. 30600122 and GuangDong Provincial Natural Science Foundation under Grant No. 06025073 .

* Email: aibq@hotmail.com

1 P. Reimann, Phys. Rep. 361, 57 (2002).

2 R. D. Astumian and P. Hanggi, Physics Today 55, 33(2002).

3 P. Hanggi, F. Marchesoni, and F. Nori, Ann. Phys. (Leipzip)14, 51 (2005).

4 R. P. Feynman, R. B. Leighton and M. Sands, The Feynman lectures on physics (Addison Wesley, Reading, MA, 1963), Vol. 1, pp.46.1-46.9.

5 J. Prost, J. F. Chauwin, L. Peliti, and A. Ajdari, Phys. Rev. Lett. 72, 2652 (1994).

6 R. D. Astumian and I. Derenyi, Phys. Rev. Lett. 86, 3859(2001); R. D. Astumian, Phys. Rev. Lett. 91, 118102 (2003).

7 I. Kosztin and K. Schulten, Phys. Rev. Lett. 93, 238102 (2004).

8 M. Rey, M. Strass, S. Kohler, P. Hanggi, and F. Sols, Phys. Rev. B 76, 085337 (2007).

9 J. F. Wambaugh, C. Reichhardt, C. J. Olson, F. Marchesoni, and F. Nori, Phys. Rev. Lett. 83, 5106 (1999).

10 S. Savelev, V. Misko, F. Marchesoni, and F. Nori, Phys. Rev. B 71, 214303 (2005).

11 J. M. Sancho and A. Gomez-Marin, Europhysics Letters 86, 40002 (2009); J. M. Sancho and A. Gomez-Marin, Phys. Rev. E 77, 031108(2008).

12 D. Reguera and J. M. Rubi, Phys. Rev. E 64, 061106 (2001); D. Reguera, G. Schmid, P. S. Burada, J. M. Rubi, P. Reimann, and P. Hanggi, Phys. Rev. Lett. 96, 130603 (2006).

13 R. Zwanzig, J. Phys. Chem. 96, 3926 (1992). 
14 P. Kalinay and J. K. Percus, Phys. Rev. E 74, 041203 (2006).

15 B. Q. Ai and L. G. Liu, Phys. Rev. E 74, 051114 (2006); B. Q. Ai, H. Z. Xie, and L. G. Liu, Phys. Rev. E 75, 061126 (2007); B. Q. Ai, L. G. Liu, J. Chem. Phys. 126, 204706 (2007).

16 A. M. Berezhkovskii, M. A. Pustovoit, and S. M. Bezrukov, J. Chem. Phys. 126, 134706 (2007).

17 D. S. Liu, R. D. Astumian, and T.Y. Tsong, J. Biol. Chem. 265, 7260 (1990); T. D. Xie, P. Marszalek, Y. D. Chen, and T.Y. Tsong, Biophys. J. 67, 1247 (1994); T. D. Xie, Y. D. Chen, P. Marszalek, and T.Y. Tsong, Biophys. J. 72, 2496 (1997).

18 S. Ramaswamy, J. Toner, and J. Prost, Phys. Rev. Lett. 84, 3494 (2000); A. E. Pelling et al., Science 305, 1147 (2004);M. Borgnia, S. Nielsen, A. Engel, and P. Agre, Annu. Rev. Biochem. $68,425(1999)$.

19 M. Switkes, C. M. Marcus, K. Campman, and A. C. Gossard, Science 283, 1905 (1999). 


\section{CAPTION LIST}

Fig. 1. Scheme of the pumping device: a spatially asymmetric tube is embedded in a finite region of length $L$ and bounded by two particle reservoirs of concentrations $\rho_{0}$ and $\rho_{1}$. The shape of the tube is determined by its radius $\omega(x)$ and the left end coordinate $x_{0}$ of the tube. The particles in the tube are powered by an unbiased external force $F(t)$.

Fig.2. Current $J$ as a function of period numbers $N$ at $x_{0}=0, a=\frac{1}{2 \pi}, b=\frac{1.5}{2 \pi}, \alpha=1 / 2$, $F_{0}=0.5, T=0.5, \rho_{0}=1$, and $\rho_{1}=1$.

Fig. 3. (a) Current $J$ as a function of $x_{0}$ for different values of $F_{0}$ at $a=\frac{1}{2 \pi}, b=\frac{1.5}{2 \pi}$, $\alpha=1 / 2, T=0.5, \rho_{0}=1$, and $\rho_{1}=1$. (b)Currents $j\left(F_{0}\right),-j\left(-F_{0}\right)$ and $J$ versus $x_{0}$ for $F_{0}=0.5$. The other parameters are the same as that in (a).

Fig. 4. The tube shape for different values of $x_{0}$. The tube is symmetric at $x_{0}=\pi / 2$ and $3 \pi / 2$. The left space is bigger than the right at $x_{0}=0$ and the right space is bigger at $x_{0}=\pi$.

Fig. 5. Ratio of concentrations as a function of $T$ at $a=\frac{1}{2 \pi}, b=\frac{1.5}{2 \pi}, \alpha=1 / 2, F_{0}=0.5$, and $x_{0}=0$.

Fig. 6. Ratio of concentrations as a function of $r_{b}$ at $a=\frac{1}{2 \pi}, \alpha=1 / 2, F_{0}=0.5, T=0.5$, and $x_{0}=0$.

Fig. 7. Ratio of concentrations as a function of $F_{0}$ at $a=\frac{1}{2 \pi}, b=\frac{1.5}{2 \pi}, \alpha=1 / 2, T=0.5$, 
and $x_{0}=0$.

Fig. 8. Current $J$ vs temperature $T$ for different values of $\rho_{1}$ at $a=\frac{1}{2 \pi}, b=\frac{1.5}{2 \pi}, \alpha=1 / 2$, $F_{0}=0.5, x_{0}=0$, and $\rho_{0}=1$.

Fig. 9. Current $J$ as a function of $r_{b}$ at $a=\frac{1}{2 \pi}, \alpha=1 / 2, F_{0}=0.5, T=0.5, x_{0}=0$, $\rho_{0}=1$, and $\rho_{1}=4$. 


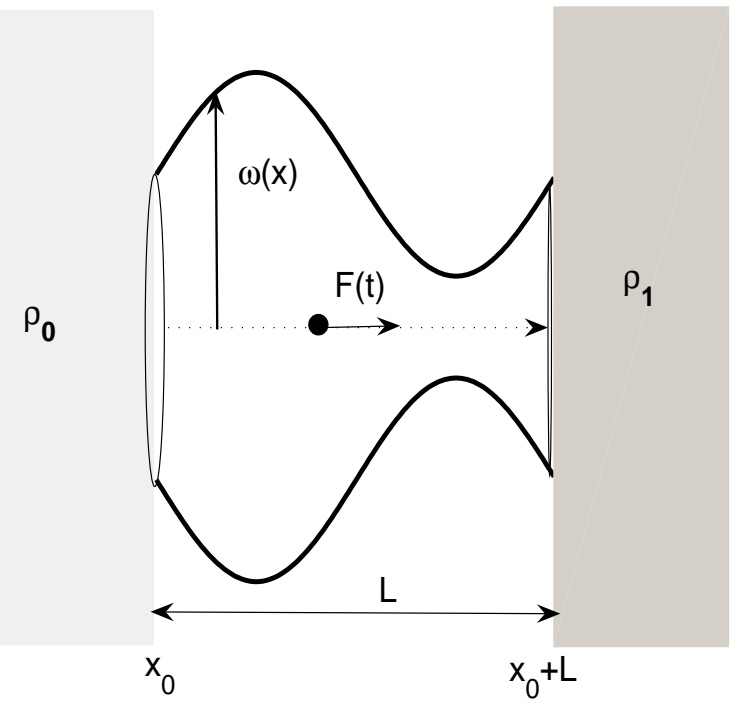

FIG. 1: 


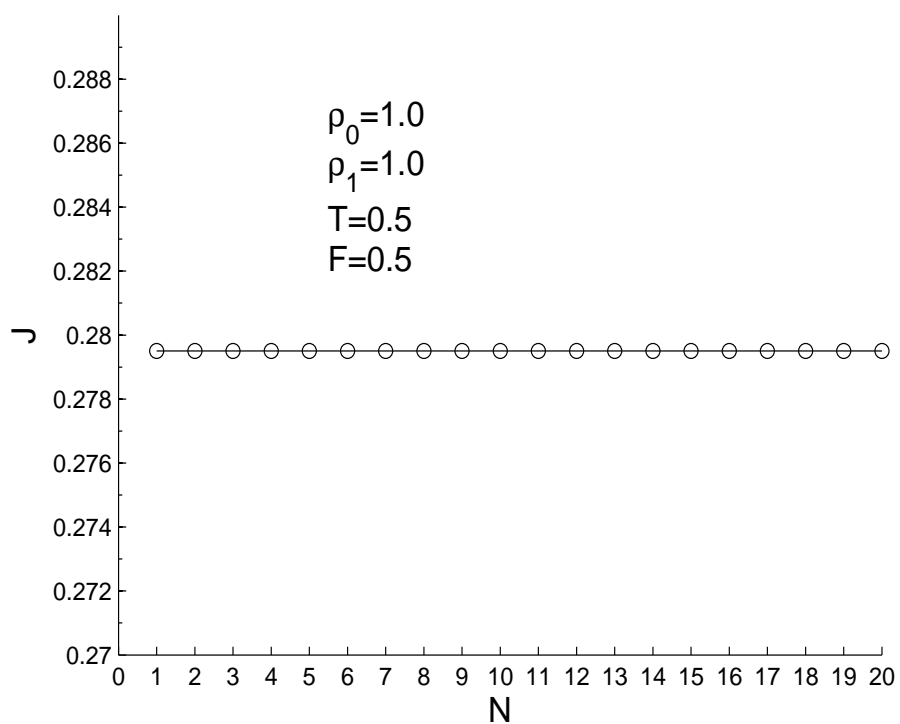

FIG. 2: 

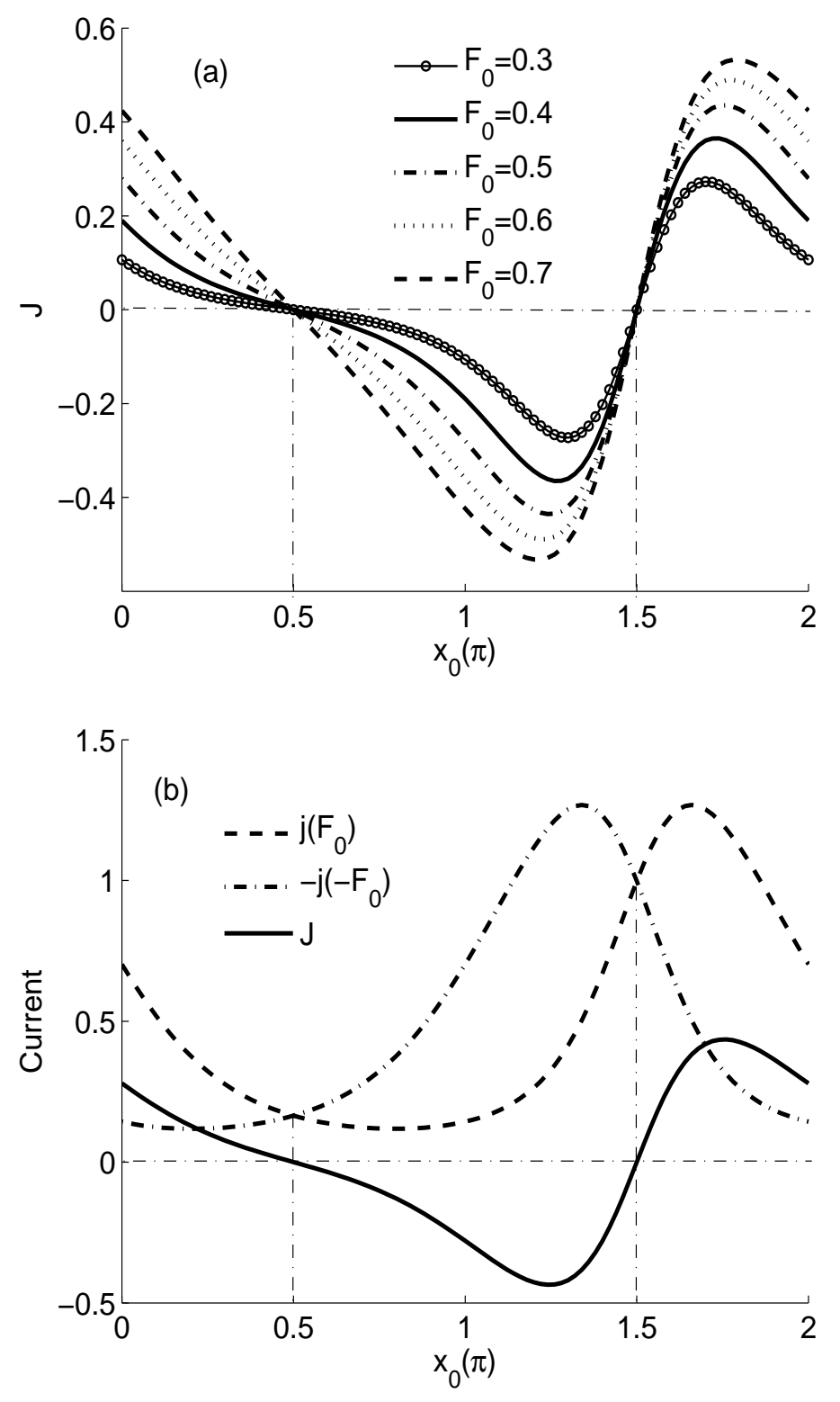

FIG. 3: 

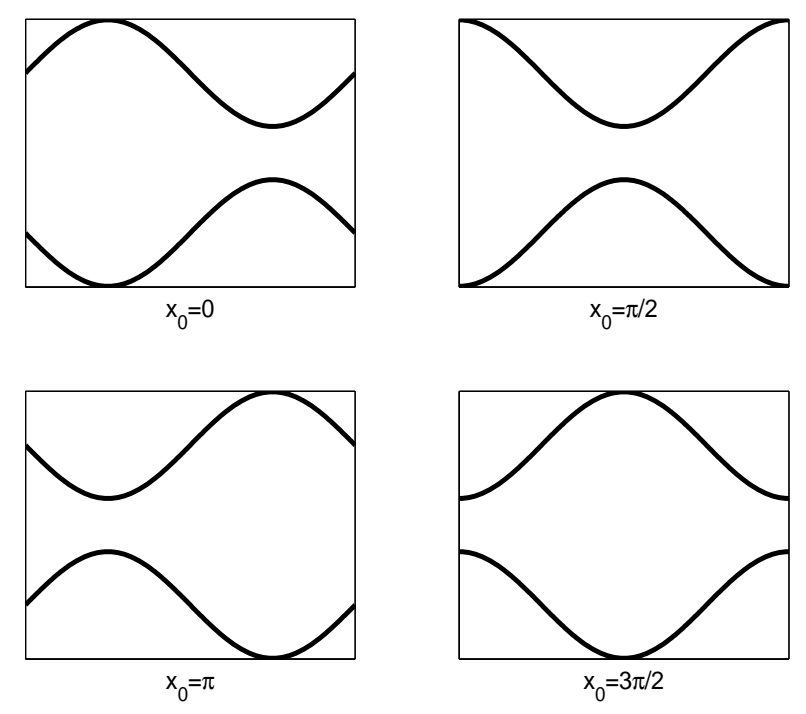

FIG. 4: 


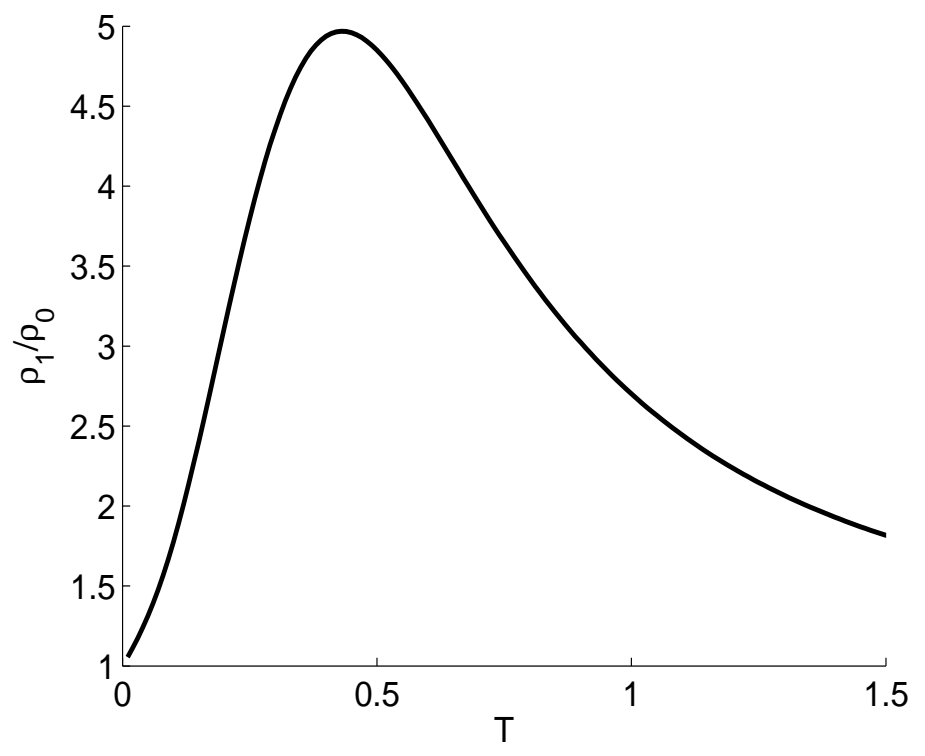

FIG. 5: 


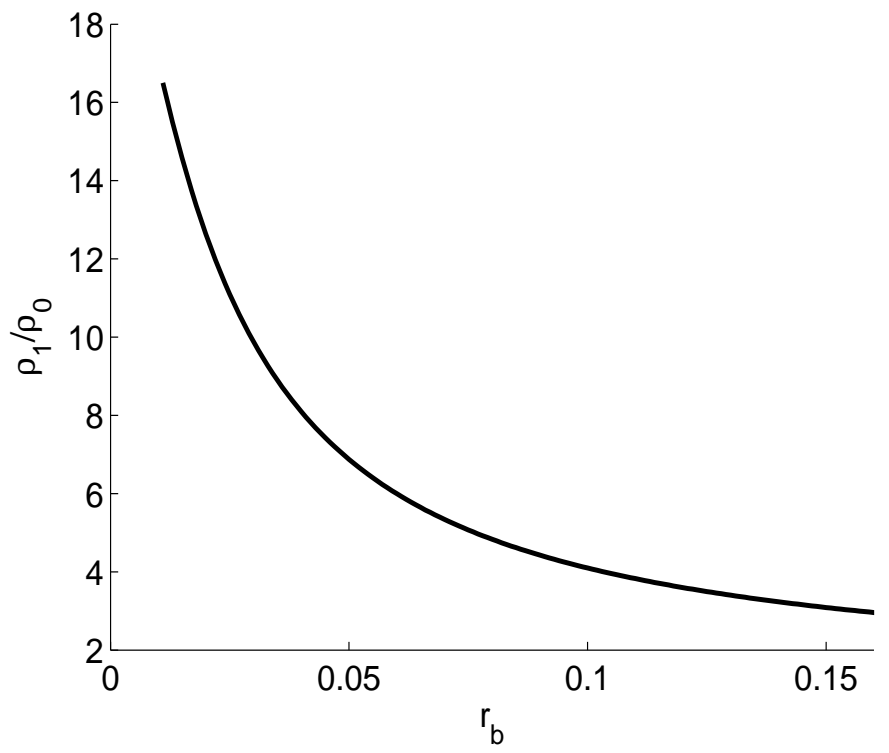

FIG. 6: 


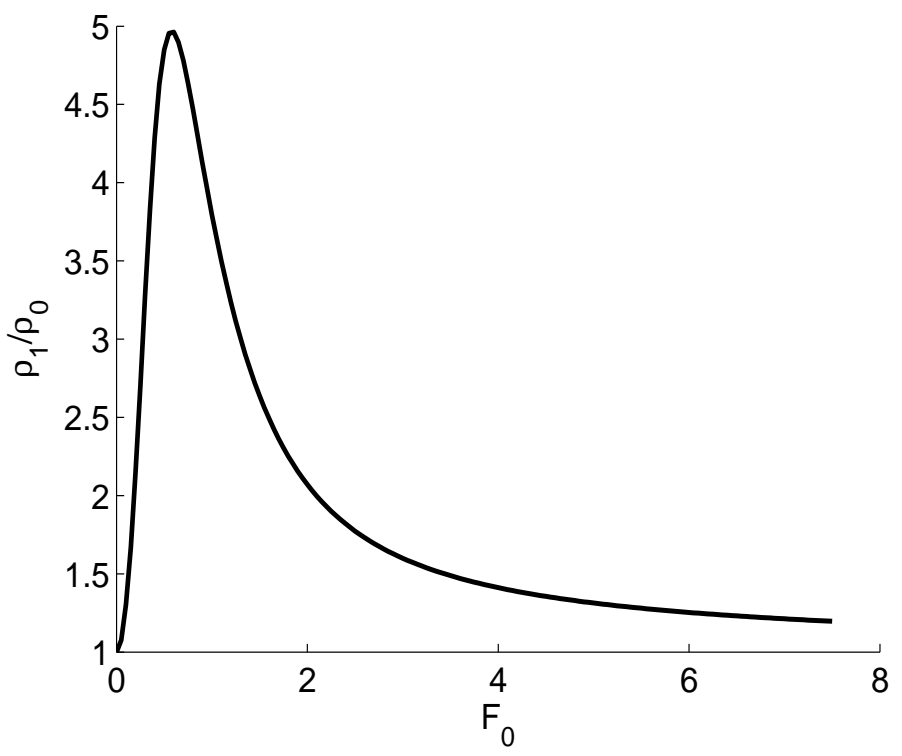

FIG. 7: 


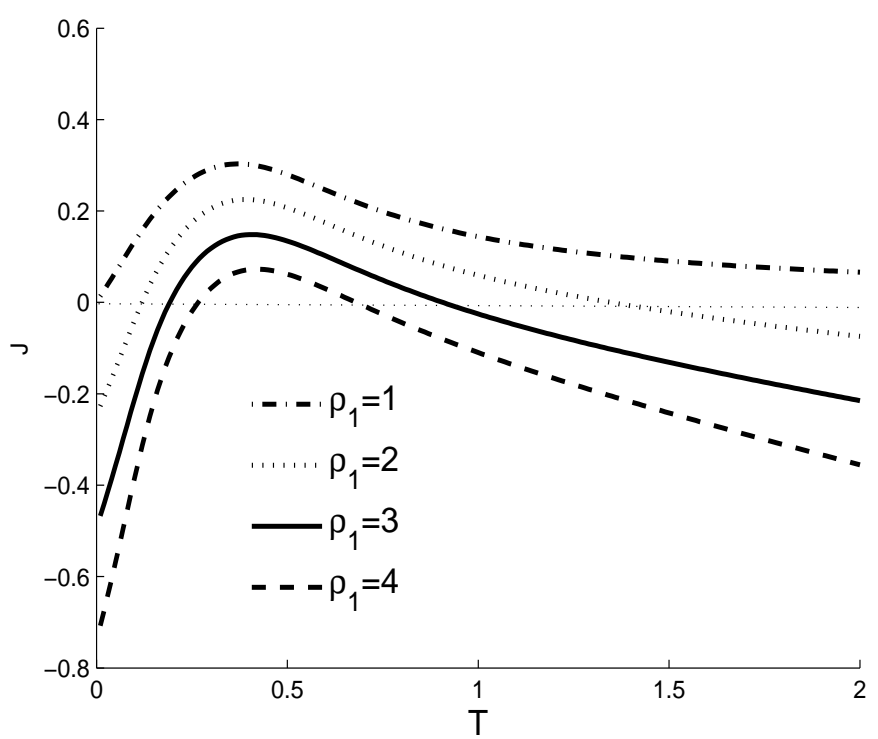

FIG. 8: 


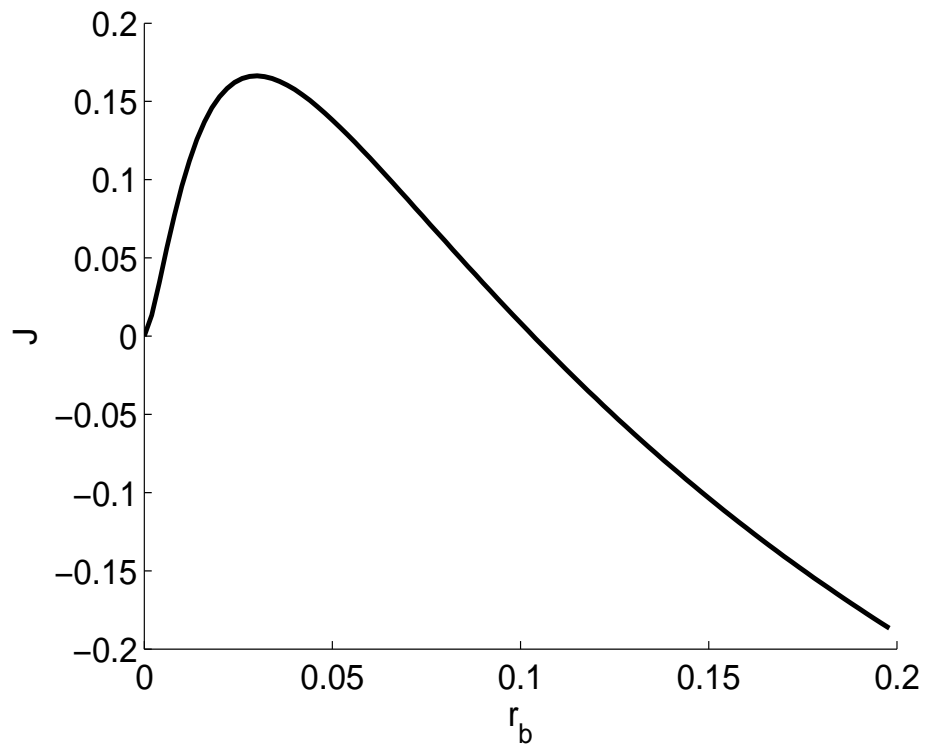

FIG. 9: 